\title{
ANALYSIS OF POSITION AND ROTATION DIRECTION OF DOUBLE STIRRER ON CHAOTIC ADVECTION BEHAVIOR
}

\author{
Sugeng Hadi Susilo \\ Department of Mechanical Engineering ${ }^{l}$ \\ sugeng.hadi@polinema.ac.id \\ Asrori Asrori \\ Department of Mechanical Engineering ${ }^{l}$ \\ asrori@polinema.ac.id \\ ${ }^{1}$ State Polytechnic of Malang \\ Jl. Soekarno Hatta No. 9, Malang, Jawa Timur, Indonesia, 65141
}

\begin{abstract}
Turbulent mixing can damage the material molecules because of turbulence. Whereas laminar mixing raises a problem when mixing is carried out on viscous liquids. The mixing mechanism using chaotic flow affects the mixing quality. The aim of the experiment was to determine the position and direction of the double stirrer chaotic mixer. The installation of a chaotic mixer uses a cylindrical tub and two different mixers consisting of a primary mixer $(P p)$ and a secondary mixer $(P S)$. Periodically rotate the container and stirrer. The center of the vessel and primary mixer are placed at the same coordinates. For $\varepsilon=4 \mathrm{~cm}(P p$ to $P s$ distance), there are three experiments, namely: vessel rotation and directional stirrer (P2S-a), vessel rotation and opposite stirrer (P2B-a), and vessel rotation, both primary and secondary stirrers are directional variations. (P2V-a). Eccentricity $7 \mathrm{~cm}$, there are also three treatments as above: one direction (P2S-b), reverse direction (P2B-b), and variation of direction (P2V-b)

The video camera recordings are processed digitally. Qualitative data show a pattern of behavior during mixing. Meanwhile, quantitative data is used to determine the level of mixing effectiveness. The results showed that the direction of rotation of the two cylinders had no effect on the effectiveness of chaotic mixing. Based on the number of initial droplets of dye, the treatment that experienced the fastest chaos was P2B-b, at $n=2$ and $r=3.5303$. The difference in the number of color droplets does not affect chaotic behavior. The highest mixing efficiency was generated by the lowest P2V-b mixing index value of 0.94. Simultaneously, the direction between the mixer and the container will provide maximum mixing efficiency. Isolated mixing areas (island) and areas of poor mixing occur because of one-way rotation and low eccentricity.
\end{abstract}

Keywords: chaotic mixer, chaotic mixing, viscous fluid, eccentricity, rotation direction, double stirrer

DOI: 10.21303/2461-4262.2021.001707

\section{Introduction}

Turbulent mixing is not always desirable because the substance consists of long molecules that will break down if turbulence occurs. Problems arise when mixing is carried out in laminar flow, especially for viscous fluids. In laminar conditions, attempts are made to make stretchers and folds to join parts that are far apart and separate adjacent parts [1]. The thicker the mixed liquid, the greater the energy required, so that the production cost is also high. Generating turbulence in this type of fluid is very uneconomical. Using propellers in viscous fluid mixing causes large motor loads and large energy consumption. Whereas mixing must occur laminar [2].

Several industries that use the mixing mechanism in their production still pay less attention to the movement pattern of the processed fluid. Such as production processes in the pharmaceutical industry, paint industry, chemical industry, oil refineries, plastic moulding, metal casting, food processing, water treatment and sewage treatment. The wrong mixing process will cause a low-quality product besides that it will also result in a waste of energy which means an additional budget [3].

In biotechnology, it uses chaos theory for mixing microorganisms and mixing in the nursery process. In mechanical engineering, the term chaos has developed into a separate study, especially in fluid mechanics. The characteristics of chaotic flow have been studied more by scientists, especially regarding fluids that are highly viscous in the laminar flow $[4,5]$. 
Chaos is a very sensitive condition because of the effect of instability in the initial conditions. Mixing that takes place in a turbulent manner is not always desirable because it will damage substances consisting of long molecules if turbulence occurs [6]. Whereas laminar flow attempts to create stretches and folds. This is useful for joining parts that are far from each other and separate parts that are close together [7].

Effective mixing results in chaotic flow. Chaos means an irregular situation with an unpredictable pattern [8]. In the mixing process, chaos is very beneficial [9]. Chaos in laminar flow can be generated by manipulating the periodic motion and shape of the system. Rotating the container and stirrer alternately or simultaneously with different speed variations is a manipulation of the movement to enrich the flow structure. Likewise, the variation in the ratio between the dimensions of the container and the stirrer could provide different mixing patterns. In the chaotic flow mixing mechanism that is not good, it can be seen the emergence of islands, which is clumps that occur in the flow path. The island will not disappear even after repeated stirring [10].

The understanding of mixing and stirring depends on the ability to create particle motion in the stream [11]. Diffusion or mixing can occur without stirring, whereas stirring does not always result in mixing. Understanding the concepts of chaotic movement and dynamic systems provides a way to generate mixtures through stirring. The further benefit of this understanding is that it is important for processes with very slow motion of molecules.

In the classical fluid mechanics system for producing chaotic turbulence, a flow created between two rotating cylinders is called a Taylor-Couette flow. This flow is sensitive to three factors, namely radius ratio $(a)$, angular velocity ratio $(\omega)$ and Reynolds number $(\mathrm{Re})$ [12].

Diffusion that occurs during chaotic motion is the main object of mixing [13]. Diffusion occurs when a drop of colour is stretched into thin lines, then separated. The separation that occurs can't be reversed (irreversible) so that the system experiences chaos.

Stretching and folding is an important concern in increasing blending effectiveness. The stretch and fold formation allow for chaos control during mixing. Strain and fold manipulation is performed to create a coherent structure. Folds determine the creation of a coherent structure in a chaotic system. A coherent structure arises when the area is fixed which is directly proportional to the width of the fold.

Strain evolution, diffusion, separation, and aggregation are the controlling factors for reactions inflow $[14,15]$. The faster the agitation system rotates, the larger the isolated area is formed. If the shear rate decreases, the unmixed area will mix with other areas. There are inundated areas as isolated mixing areas (IMR) [11, 12]. IMR is formed as a function of the fold distance from the initial position of the colour and the rotation of the container.

Therefore, research related to chaos stirring was carried out, to detect and predict IMR, randomness, exploitation and control of IMR using experiments and computations. The mechanism that plays a role in this stirring is a cylindrical container with a double rotating stirring tube. Once the mixing patterns of paints and dyes are observed, the characteristics of the best-mixed conditions can be used for other similar processes.

\section{Materials and methods}

The equipment in this research consisted of a chaotic double mixer, Sony camcorder handycamp, PLC, tachometer, $6 \mathrm{~A}$ adapter and a computer. Fig. 1 shows a mixer with a double mixer. This machine consists of a cylindrical container and two stirrers. Rotating the container and stirrer each using a DC electric motor $(25 \mathrm{~V})$. Primary $\left(R_{p}=19 \mathrm{~mm}\right)$ and secondary $\left(R_{S}=8.5 \mathrm{~mm}\right)$ stirrers. The container is made of transparent cylindrical plastic with a radius of $R_{w}=80 \mathrm{~mm}$ and a height of $170 \mathrm{~mm}$. Container rotation $\left(n_{w}\right)=13 \mathrm{rpm}$, primary mixer rotation $\left(n_{p}\right)=10 \mathrm{rpm}$ and secondary mixer $\left(n_{s}\right)=22.3 \mathrm{rpm}$. The angular velocity of the primary mixer $W_{p}=1800^{\circ}$ and container $W_{w}=360^{\circ}$. So that the ratio of angular velocity $\omega=W_{p} / W_{w}=5$ and the radius ratio $a=\left(R_{p}+R_{s}\right) / R_{w}=0.34$ is obtained.

The primary mixer is installed in the centre of the container, while the eccentricity, the secondary mixer, is varied. The direction of rotation of the stirrer is made to vary with the direction of rotation of the container and is carried out alternately continuously. The test fluid used is a mixture of wall paint and water with a volume ratio of $1: 1$. The dye uses a mixture of paint and water (1:3). 




Fig. 1. Experimental setup

Measurement of the viscosity of test fluids and dyes is done using a Brookfield Viscometer manufactured by Brookfield Engineering Laboratories, Inc. The viscometer used is the RV model which has eight spindles and eight levels of speed. The test specimen viscosity value is calculated by multiplying the number displayed on the screen by the multiplier of the spindle used. Minimum measurement values are obtained using high and large speed spindles, while maximum values are obtained using low and small speed spindles. Measurements using the same spindle with variations in velocity were carried out to determine fluid rheology.

The experimental design can be seen in Fig. 2. The primary stirrer is at the centre point of the container. At a distance of the two stirrers of $4 \mathrm{~cm}(\varepsilon=4 \mathrm{~cm})$, there are three designs: the second cycle of the stirrer with a unidirectional container $(\mathrm{P} 2 \mathrm{~S}-\mathrm{a})$, the second rotation of the stirrer with the container in the opposite direction (P2B-a) and the rotation between the stirrer and the container varies in direction $(\mathrm{P} 2 \mathrm{~V}-\mathrm{a})$. At $7 \mathrm{~cm}(\varepsilon)$ eccentricity, there are three treatments, namely unidirectional (P2S-b), opposite direction (P2B-b) and variation of direction (P2V-b).



Fig. 2. Combined experimental designs

The mixing process was recorded using a Sony Handy camp camera. Recordings are stored in the .mpeg file format, then extracted per segment into JPG format, so that the results can be analyzed by a computer. Observations were made on the fluid surface (2-D system). The image used for analysis is a picture of each mixing cycle, namely when the container rotates $360^{\circ}$ and the primary stirrer rotates $180^{\circ}$. Observations were made for up to 60 rotation periods. It can be seen from the mixing time and the percentage of a liquid mixture.

Analysis of the distribution and percentage of colour mixing was carried out using Adobe Photoshop software version 7.0. Image colours are changed to black and white for easy analysis using the Image Adjustments Threshold feature with a Threshold level of 166. Colours smaller than 166 will be white and those larger than 166 will be black. There is no grey colour. The dye traces are black, and the liquid is white. The percentage of colour is calculated using the Histogram facility which contains black percentage information. The percentile is the ratio between the number of black pixels and the total image pixels. 


\section{Results and discussion}

The research results show that the fluid and dye test have the same properties, namely non-Newtonian. Fig. 3, 4 shows the relationship between viscosity and spindle rotation (rpm). Fig. 3, 4 describes the properties of the test fluid and dye, it shows a decrease in the value of viscosity with increasing rpm. In the discussion of fluid rheology three parameters must be known, namely viscosity, shear stress $(\tau)$ and shear rate $(g)$ [16]. The two figures below also indirectly show the relationship between viscosity and shear rate because the Brookfield viscometer assumes that the spindle rotation speed is proportional to the fluid shear rate.

Fig. 4 shows that the viscosity decreases with increasing shear rate. So, that from these characteristics, the test fluid and dye have the same type, namely the non-Newtonian time-independent fluid, which is the pseudoplastic (shear-thinning) type.

While the equations of motion for viscous fluids use the Navier-Stokes nonlinear partial differential equation (NLPDE).

$$
\frac{\partial v}{\partial t}+(v \cdot \operatorname{grad}) v=-\operatorname{grad} \frac{(P)}{\rho} \operatorname{grad}(\phi)+\frac{\eta}{\rho} \operatorname{lapl}(v)
$$

where $P$ is the pressure, $v(r, t)$ is the velocity of the fluid at point $r, \phi(r)$ is the gravitational potential, $\rho$ is the density of the fluid, and $\eta$ is the viscosity. This equation relates pressure, fluid velocity, density and viscosity [17]. The Reynolds number for laminar flow is very small $(\operatorname{Re}=v L \rho / \eta \ll 1)$, where $\mathrm{L}$ is the length of the flow and $\mathrm{v}$ is the velocity of the fluid. So that the approach to equation (1) can be done with linear partial differential equations (LPDE). All fluid volume elements represent well-defined paths $r=r(t)$. It is because the number of volume elements $\delta \mathrm{V}$ is not limited. The LPDE has infinite degrees of freedom, and this is a characteristic of PDE. For $\operatorname{Re} \gg 1$ the nonlinear effect becomes dominant. So that the flux becomes irregular. It is characterized by irregular trajectories of fluid elements and forming eddies, ripples and circles. In addition, there is a kind of order in a chaotic flow which is described as fractal. Based on equation 1, the viscosity of the fluid affects the flow velocity, in that it will slow down the rotation of the stirrer.

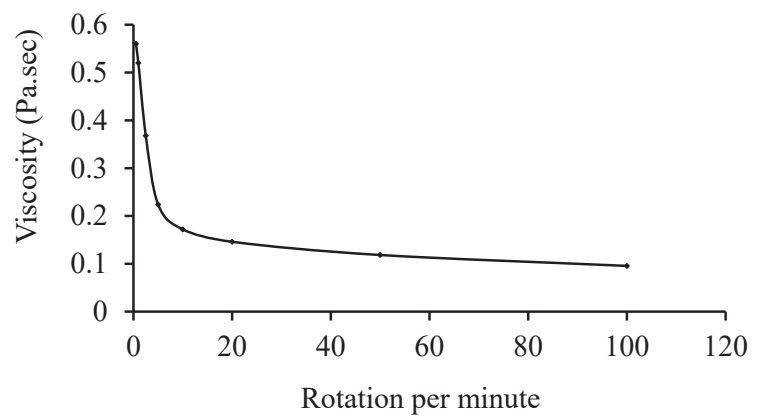

Fig. 3. The relationship between the viscosity and rpm of the test fluid

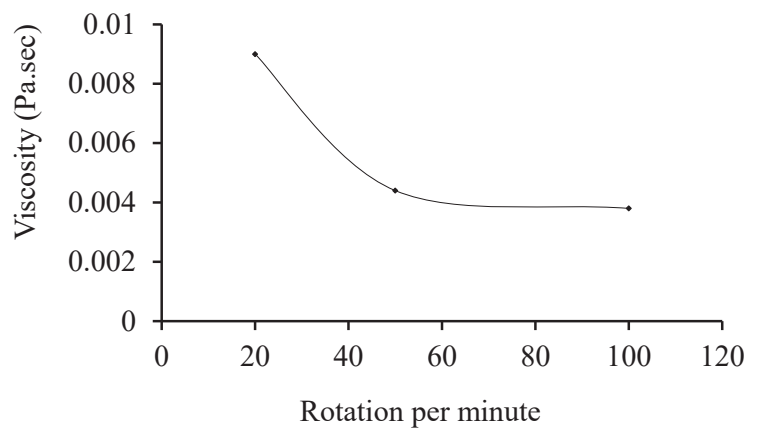

Fig. 4. Relationship between viscosity and rpm of dye 
The results of this research indicate the viscosity of the test fluid at $13 \mathrm{rpm}$ and $10 \mathrm{rpm}$ primary stirrer is $0.160 \mathrm{~Pa} \cdot \mathrm{sec}$ and $0.172 \mathrm{~Pa} \cdot \mathrm{sec}$, respectively. Whereas for the dye test the viscosity value was detected at a speed of $20 \mathrm{rpm}$ so that for speeds less than $20 \mathrm{rpm}$ the viscosity value was $0.009 \mathrm{~Pa} \cdot \mathrm{sec}$. The comparison of the viscosity of the dye with the test fluid in the two rounds mentioned above is $p 13=0.06$ and $p 10=0.057$. From the comparison of the viscosity of the dye and the test fluid, it can be seen that the value of $p \ll 1$. The dye stretches more easily when $p \gg 1$ [18]. In this study, the Reynold number $(\mathrm{Re}) \gg 110.67$ is obtained. With a low Reynolds number, the stirring model is expected to produce chaotic advection for viscous fluids that are non-Newtonian.

\section{1. Concentric cylindrical flow pattern}

The results show that under certain conditions, many use an eccentric cylinder mechanism rather than a concentric cylinder. Fig. 5 shows the results of the study using two concentric cylinders, (top) the container and unidirectional stirrer (P1S-o) and (bottom) both unidirectional cylinders (P1B-o).

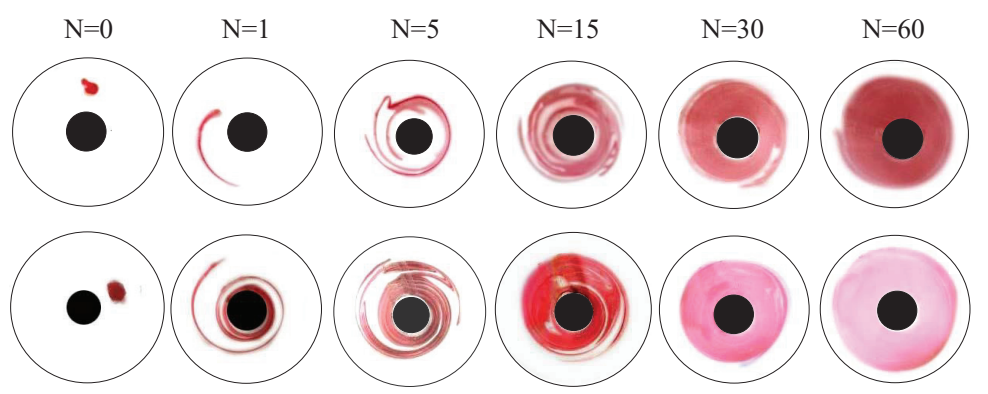

Fig. 5. The tracking pattern on the concentric cylinder during the $N$-period

Fig. 5 indicates that the distribution of colours takes a long time (up to a period of $N=60$ ), the colours do not occupy the entire area of the container. At $N=5$, the colour trajectory only surrounds the inner cylinder, and in the following period, the colour trajectory is relatively stable despite the colour distribution. When the flow pattern is stable, tend to result in inefficient mixing.

The stable trajectory is caused by the trajectory area of the fluid elements between two concentric cylinders having the same shear rate and viscosity so that the dye flow strain occurs. Fig. 6 shows the colour growth rate which tends to be linear until the $60^{\text {th }}$ period.

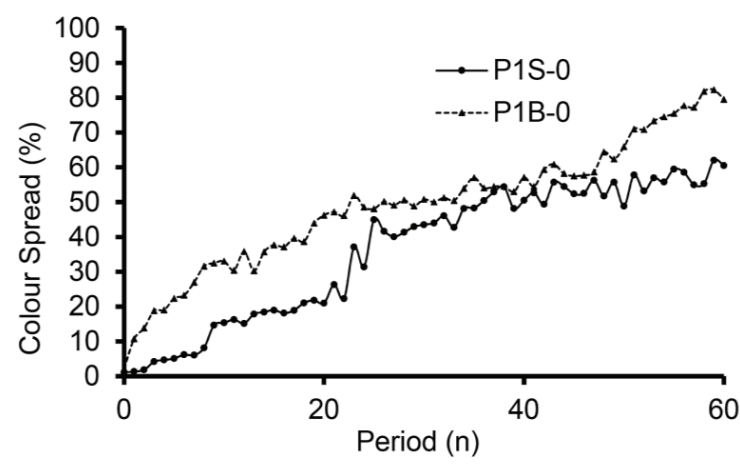

Fig. 6. Distribution of dye to period in concentric cylinders, P1S-o and P1B-o

The variation in the direction of rotation of the two cylinders (P1B-o) tends to produce the same conditions as P1S-o. This means that the difference in the direction of rotation of the two concentric cylinders does not have a significant effect on the effectiveness of the mixing.

\section{2. Identification of chaos mixing behavior}

The results of the research on the mechanism of chaotic mixer with double stirrers then describe the relationship of colour distribution to period $(N)$, see Fig. 7. 


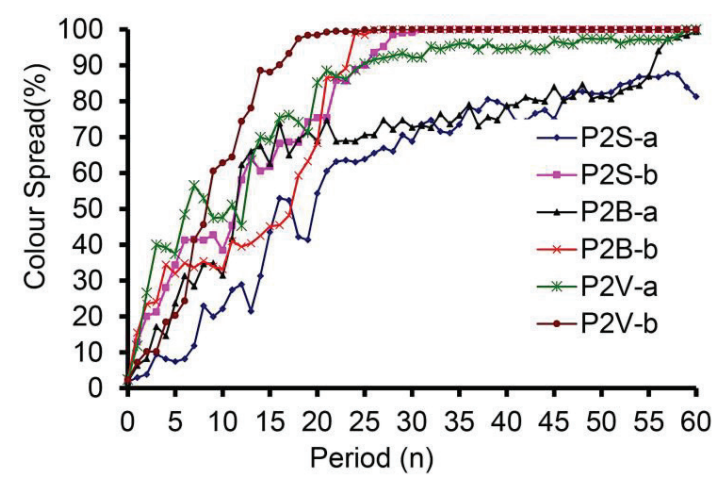

Fig. 7. The relationship between colour distribution (\%) and period (n)

Fig. 7 shows that double stirrer treatment resulted in exponential colour growth. Thus, it can be proven that the stirring design mechanism used in this study is capable of producing chaotic mixing patterns.

\section{3. Visually chaotic behavior}

The experimental results show the known chaotic flow by visualizing the flow patterns that are formed. Fig. 8 shows chaotic behaviour, the growth mechanism of the drop of colour flow (initial conditions) into a chaotic pattern.

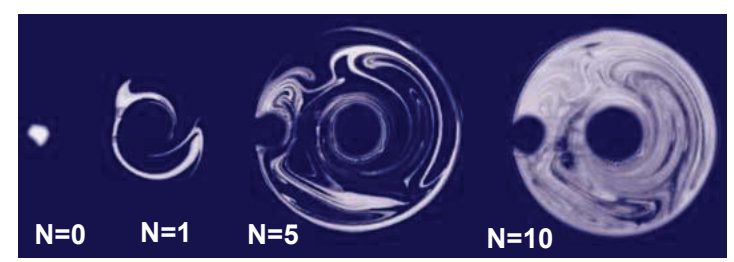

Fig. 8. The behaviour of the chaotic flow growth of $\mathrm{P} 2 \mathrm{~V}-\mathrm{b}$ treatment until the $10^{\text {th }}$ period

Fig. 8 indicates that a drop of colour undergoes a stretching, bending, and folding mecha$\operatorname{nism}(N=1)$. The same mechanism repeatedly occurs to form a thin film that spreads throughout the container $(N=10)$. The above phenomenon indicates a chaotic mixing process.

Fig. 9 illustrates the periodic increase in the stretch and crease of the paint dough flow line. The periodic stretches and folds cause the colour to pass through several trails allowing enough streaks of colour to grow further. The growth of one colour line (cycle-1) into two lines is indicated by branching at $r=2.9$. Each colour line (cycle-2) then forms a new colour line at $r=3.4$. The $3^{\text {rd }}$ cycle forms more lines until they intersect each other at $r=3.65$. So that for $r>3.65$ the colour lines that are formed are very many and overlap each other or it is called chaos.

The research results showed a chaotic mixing flow pattern, as shown in Fig. 10.

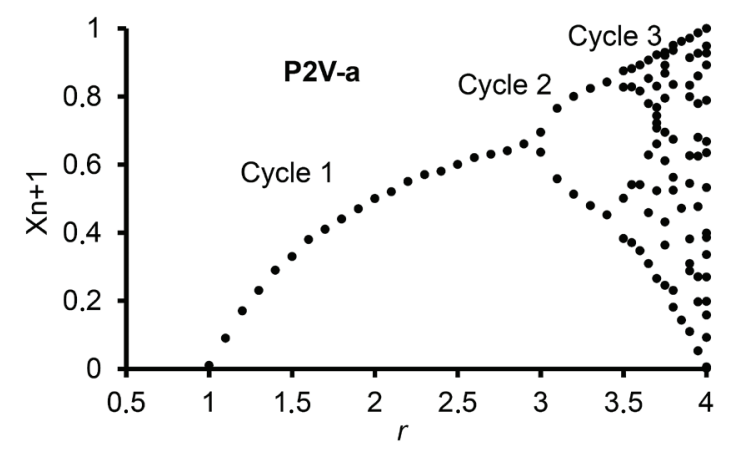

Fig. 9. The relationship between growth rate, $r$, to the colour distribution, $X n+1$, in the form of a bifurcation diagram for $\mathrm{P} 2 \mathrm{~V}$-a treatment 


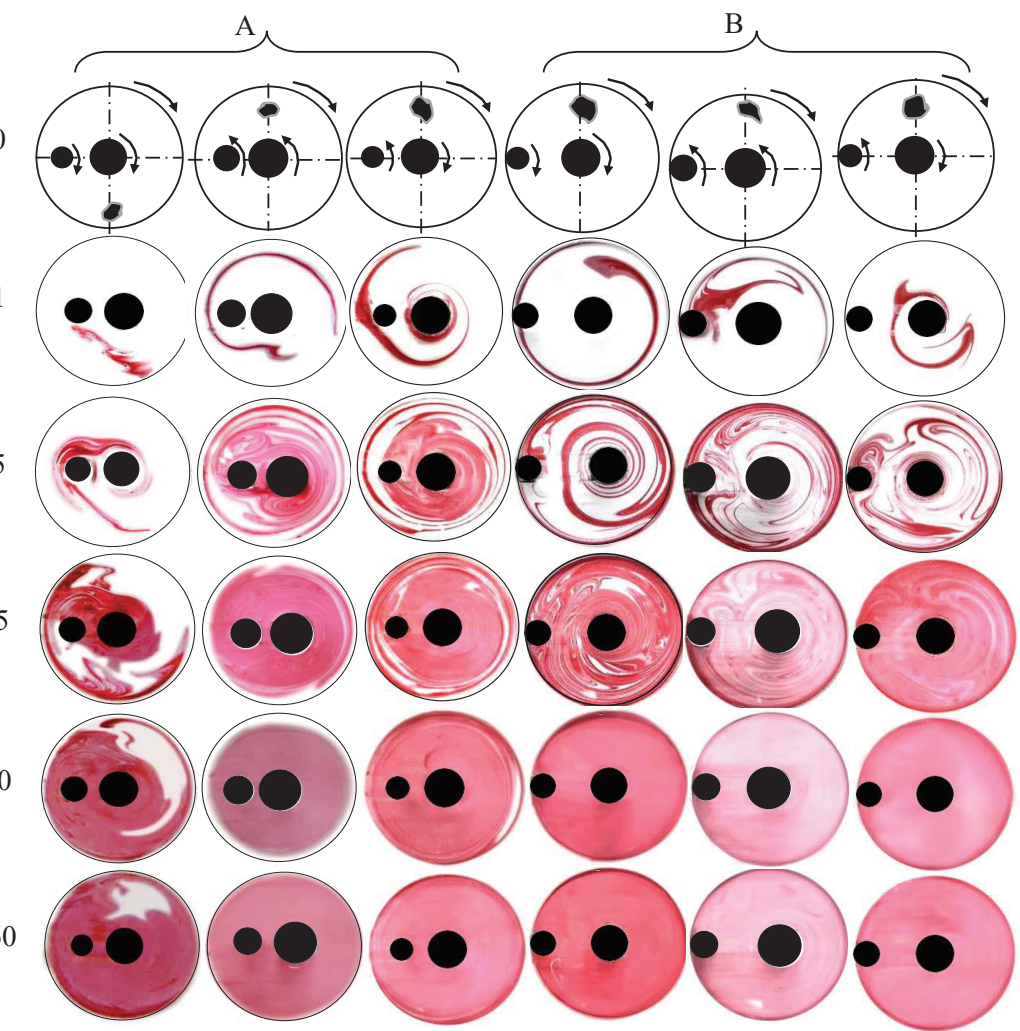

Fig. 10. Dye flow pattern from period 0 to 60 (A) for $\varepsilon=4 \mathrm{~cm}$, from left to right P2S-a, P2B-a, P2V-a. (B) for $\varepsilon=7 \mathrm{~cm}$, from left to right P2S-b, P2B-b, P2V-b. All treatments at $\omega=5, a=0.34$

Chaotic behaviour develops rapidly if it occurs in the periodic (intermittent) flow region. Variations in the direction of rotation are needed to determine the most efficient treatment and produce homogeneous stirring. The mixing of two fluids is formed due to advection which results in particle deformation. So, that variations in direction and speed in moving fluid are very important.

In the treatment with stirring rotation in the direction of the container, it tends to only extend the colour stretch and create a thick layer of colour so that the colour is difficult to get out of the wider path. This is because the flow between two unidirectional cylinders will produce a flow line path to the equilibrium point (fixed point). So that the dye fluid elements are not able to make divergent pathways. The stability of flow in a laminar viscous fluid will cause a thick layer of flow. So, that the breakdown of this particle layer takes a long time.

In the first period where the direction of rotation of the stirrer is opposite to the container, the dye flow line undergoes a stretching process. The folding process around the mixer follows the bending process. At the same time, a mixer, whose rotation varies with the container, quickly forms a chaotic region. Meanwhile, mixing in different directions between the two mixers and the container results in a fast mixing mechanism. The direction of rotation between the mixer and this container will determine the chaotic flow pattern. This chaotic flow will produce a layer structure consisting of thousands of lines that have a wide thickness distribution. The flow tracker rapidly extends into the layers of the system continues as the period increases. The layers become thin as a result of the complex repetitive processes of stretching, spreading and folding mechanisms.

The double stirrer eccentricity aims to expand the shear plane. It speeds up the stretching, contraction and folding mechanisms of the dye. Once the mess is formed, the dye is rapidly restretched, broken and dispersed into a thin flow layer.

The eccentricity variation between primary and secondary stirrers also aims to break up isolated areas so that homogeneous mixing occurs. Stirring using an eccentric two-cylinder mecha- 
nism (container and stirrer) causes the whole mixing process to have a large fold. Meanwhile, the large fold pattern that repeats itself at a constant time makes it difficult to spread or break, resulting in isolated areas or islands.

This is formed where there is no meeting between the shear rate of the stirrer and the shear rate of the container. The island structure prevents mixing. In this study, isolated areas occurred in treatment with an eccentricity of $3 \mathrm{~cm}$ (P2S-a and P2V-a), see Fig. 11.

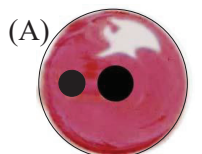

(B)

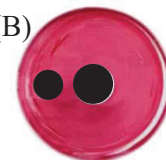

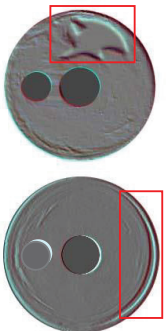

Fig. 11. Isolated area pattern (island), highlighted in the box with the relief image to the right:

$$
\mathrm{A}-\mathrm{P} 2 \mathrm{~S}-\mathrm{a}, N=60 ; \mathrm{B}-\mathrm{P} 2 \mathrm{~V}-\mathrm{a}, N=25
$$

Apart from the formation of islands, there are also areas of poor mixing, usually forming around the walls of the container. This phenomenon occurs in the flow between two concentric cylinders. The stirrer which is located in the concentric shear plane is only limited around the complainant so that up to a certain radius the colour flow lines form a stable pattern.

\section{Conclusion}

1. The variation in the direction of rotation of the two concentric cylinders does not have a significant effect on the effectiveness of the colour distribution of the chaotic mixing mechanism. Based on the number of initial droplets of dye, the treatment that experienced the fastest chaos was P2B-b, at $n=2$ and $r=3.5303$. The difference in the number of colour droplets in this experiment did not affect chaotic behaviour, it was seen that the r-value was relatively the same. The highest mixing efficiency until the $60^{\text {th }}$ period was produced by the lowest mixing index value, namely the P2V-b treatment of 0.94 .

2. Variation in direction between stirrer and container and increased eccentricity between stirrers will provide maximum mixing efficiency. Isolated mixing area and poor mixing area are due to unidirectional rotation and small eccentricity.

\section{Acknowledgments}

We express our gratitude to the Department of Mechanical Engineering, State Polytechnic of Malang, to provide equipment support and be more mature in conducting an analysis.

\section{References}

[1] Study on energy efficiency and energy saving potential in industry and on possible policy mechanisms. Contract No. ENER/ C3/2012-439/S12.666002. Available at: https://ec.europa.eu/energy/sites/ener/files/documents/151201\%20DG\%20ENER\%20 Industrial\%20EE\%20study\%20-\%20final\%20report_clean_stc.pdf

[2] Kreczak, H. E. (2019). Rates of mixing in models of fluid devices with discontinuities. University of Leeds. Available: https:// etheses.whiterose.ac.uk/26449/2/thesis-HKreczak.pdf

[3] Topics on environmental and physical geodesy (2014). Dept. Fisica Aplicada UPC, Barcelona Tech. Available at: https:// upcommons.upc.edu/bitstream/handle/2117/81242/Topics_in_Environmental_and_Physical_Geo\%28Redondo\%29.pdf?sequence $=1 \&$ isAllowed $=\mathrm{y}$

[4] Sajid, M. (2013). Recent Developments on Chaos in Mechanical Systems. International Conference on Mechanical and Industrial Engineering, 218-221. Available at: https://www.researchgate.net/publication/262524523_Recent_Developments_on Chaos_in_Mechanical_Systems

[5] Dayan, N. (Ed.) (2016). Handbook of Formulating Dermal Applications. John Wiley \& Sons, Inc. doi: https://doi.org/10.1002/ 9781119364221 
[6] Sivák, P., Delyová, I., Bocko, J., Šarloši, J. (2014). Some Methods of Analysis of Chaos in Mechanical Systems. American Journal of Mechanical Engineering, 2 (7), 199-203. doi: https://doi.org/10.12691/ajme-2-7-6

[7] Speetjens, M., Metcalfe, G., Rudman, M. (2019). Lagrangian transport and chaotic advection in three-dimensional laminar flows. arXiv.org. Available at: https://arxiv.org/pdf/1904.07580.pdf

[8] Gouillart, E. (2008). Chaotic mixing by rod-stirring devices in open and closed flows. HAL. Available at: https:// tel.archives-ouvertes.fr/tel-00204109/document

[9] Aref, H., Blake, J. R., Budišić, M., Cardoso, S. S. S., Cartwright, J. H. E., Clercx, H. J. H. et. al. (2017). Frontiers of chaotic advection. Reviews of Modern Physics, 89 (2). doi: https://doi.org/10.1103/revmodphys.89.025007

[10] Gilpin, W. (2018). Cryptographic hashing using chaotic hydrodynamics. Proceedings of the National Academy of Sciences, 115 (19), 4869-4874. doi: https://doi.org/10.1073/pnas.1721852115

[11] Furukawa, H., Kato, Y., Inoue, Y., Kato, T., Tada, Y., Hashimoto, S. (2012). Correlation of Power Consumption for Several Kinds of Mixing Impellers. International Journal of Chemical Engineering, 2012, 1-6. doi: https://doi.org/10.1155/2012/106496

[12] Kusumastuti, A., Anis, S., Najibulloh, G. M. (2019). Taylor-Couette Column for Emulsion Liquid Membrane System: Characterisation Study. Jurnal Bahan Alam Terbarukan, 8 (1), 22-27. doi: https://doi.org/10.15294/jbat.v8i1.20162

[13] Susilo, S. H., Suparman, S., Mardiana, D., Hamidi, N. (2016). The Effect of Velocity Ratio Study on Microchannel Hydrodynamics Focused of Mixing Glycerol Nitration Reaction. Periodica Polytechnica Mechanical Engineering, 60 (4), $228-232$. doi: https://doi.org/10.3311/ppme.8894

[14] Shirmohammadi, F., Tohidi, A. (2019). Experimental and numerical analysis of chaotic advection as an efficient approach to maximize homogeneous laminar mixing in a batch mixer. Brazilian Journal of Chemical Engineering, 36 (4), $1463-1473$. doi: https://doi.org/10.1590/0104-6632.20190364s20180559

[15] Dunn, M. J. (2008). Finite-Rate Chemistry Effects in Turbulent Premixed Combustion. University of Sydney. Available at: https://ses.library.usyd.edu.au/handle/2123/5782

[16] Christidis, G. E. (2010). Industrial Clays. Advances in the Characterization of Industrial Minerals, 341-414. doi: https:// doi.org/10.1180/emu-notes.9.9

[17] Li, Y. (2002). Chaos in partial differential equations. Contemporary Mathematics, 93-115. doi: https://doi.org/10.1090/ conm/301/05160

[18] Van Gils, D. P. M., Huisman, S. G., Grossmann, S., Sun, C., Lohse, D. (2012). Optimal Taylor-Couette turbulence. Journal of Fluid Mechanics, 706, 118-149. doi: https://doi.org/10.1017/jfm.2012.236

How to cite: Susilo, S. H., Asrori, A. (2021). Analysis of position and rotation direction of double stirrer on chaotic advection behavior. EUREKA: Physics and Engineering, 2, 78-86. doi: https://doi.org/10.21303/2461-4262.2021.001707 